\title{
A phase II trial of preoperative chemotherapy with epirubicin, cisplatin and capecitabine for patients with localised gastro-oesophageal junctional adenocarcinoma
}

\section{N Starling', A Okines', D Cunningham*,', W Allum², A Wotherspoon ${ }^{3}$, M Benson', J Thompson', J Thomas', G Brown ${ }^{4}$, A Riddell ${ }^{4}$, F Stavridi', S Ashley ${ }^{5}$, J Oates' and I Chau'}

'Department of Medicine, Royal Marsden Hospital NHS Foundation Trust, Surrey and London, UK; ${ }^{2}$ Department of Surgery, Royal Marsden Hospital NHS Foundation Trust, Surrey and London, UK; ${ }^{3}$ Department of Histopathology, Royal Marsden Hospital NHS Foundation Trust, Surrey and London, UK; ${ }^{4}$ Department of Radiology, Royal Marsden Hospital NHS Foundation Trust, Surrey and London, UK; ${ }^{5}$ Department of Computing and Statistics, Royal Marsden Hospital NHS Foundation Trust, Surrey and London, UK

Preoperative cisplatin/fluorouracil is used for the treatment of localised oesophageal carcinoma. This phase II study aimed to assess the efficacy and safety of administering preoperative epirubicin/cisplatin/capecitabine (ECX). Patients with stage II or III oesophageal/ gastro-oesophageal junctional adenocarcinoma from one institution received 4 cycles of ECX (epirubicin $50 \mathrm{mg} \mathrm{m}^{-2}$ day I, cisplatin $60 \mathrm{mg} \mathrm{m}^{-2}$ day I, capecitabine $625 \mathrm{mg} \mathrm{m}^{-2}$ b.i.d. daily) followed by surgery. The primary end point was the pathological complete response $(\mathrm{pCR})$ rate based on a Simon two-stage design. Secondary end points included overall and progression-free survival (OS/ PFS). Thirty-four patients were recruited: median age 60 years (range 4I -8I), 9I\% male, 97\% PS 0/I, 80\% T3, 68\% NI. Thirty-one patients completed four ECX cycles. Grade 3/4 toxicities $\geqslant 5 \%$ included neutropenia (62\%), hand-foot syndrome (I 5\%) and nausea/ vomiting (9\%). Thirteen out of $28(46 \%)$ evaluable patients responded to chemotherapy by EUS ( $\geqslant 30 \%$ reduction in maximal tumour thickness). Twenty-six out of 34 (76\%) patients underwent resection (R0 =73\%, RI =27\%). Post-operatively, two patients died within 60 days of surgery. The pCR rate was 5.9\% (95\% Cl 0-14\%) in the intent-to-treat population. According to the statistical design, this prompted early study termination. However, with a median follow-up of 34 months the median OS and I- and 2-year survival rates were 17 months, 67 and 39\% respectively. Median PFS was 13 months. Of the 14 relapsed patients, 10 presented with distant metastases. Preoperative ECX is feasible and well tolerated. Although associated with a low pCR rate, survival with ECX was comparable with published studies suggesting that PCR may not correlate with satisfactory outcome from preoperative chemotherapy for localised oesophageal adenocarcinoma.

British Journal of Cancer (2009) 1 00, I725- 1730. doi:I0.1038/sj.bjc.6605070 www.bjcancer.com

Published online 12 May 2009

(c) 2009 Cancer Research UK

Keywords: oesophageal adenocarcinoma; preoperative chemotherapy; pathological complete response

Although the prevalence of distal gastric cancer has declined, the world-wide incidence of distal oesophageal and gastro-oesophageal junctional (GOJ) adenocarcinoma is increasing (Blot et al, 1991; McCann, 1999; Pera et al, 2005). It is estimated that over 462000 new cases of oesophageal cancer were diagnosed world-wide in 2002 accounting for 386000 deaths (Parkin et al, 2005). For patients who present with localised oesophageal adenocarcinoma surgery remains the cornerstone of treatment, potentially improving long-term survival. However, despite radical resection, 5-year survival rates range from only 15-39\% (Malthaner et al, 2006).

Several surgical adjuncts have been investigated to improve prognosis. Preoperative chemotherapy offers the potential for tumour downstaging, early symptom improvement, enhanced

*Correspondence: Professor D Cunningham, Department of Medicine, Royal Marsden Hospital, Downs Road, Sutton, Surrey SM2 5PT, UK; E-mail: david.cunningham@rmh.nhs.uk

Received 7 November 2008; revised 30 March 2009; accepted 6 April 2009; published online 12 May 2009 resectability, the demonstration of in vivo chemosensitivity, the early treatment of micrometastatic disease and improvement in disease-free and overall survival (OS). This approach is supported by the results of the Medical Research Council (MRC) randomised trial of 802 patients with localised oesophageal cancer, which showed a significant improvement in 2-year survival for preoperative cisplatin/fluorouracil compared with the surgery alone (43 vs 34\%, respectively) with both the adenocarcinoma and squamous carcinoma subgroups appearing to benefit (MRC, 2002). With a longer median follow-up of 6 years, the 5-year survival for the preoperative chemotherapy $v s$ surgery alone arms was still significantly better (23 vs 17\%) (Allum et al, 2008). In contrast, the US Intergroup 113 trial of 440 patients failed to show an advantage for peri-operative cisplatin/fluorouracil $v s$ surgery alone in oesophageal cancer (2-year survivals of 35 vs 37\%, respectively) leading to variation in the uptake of this practice (Kelsen et al, 1998). However, in a separately published subgroup analysis, responders to preoperative chemotherapy (as gauged by barium oesophagrams) had a significantly better outcome than nonresponders (Kelsen et al, 2007). In a systematic review of 11 
randomised studies involving 2019 patients with oesophageal carcinoma, preoperative cisplatin-based chemotherapy appeared to confer a survival advantage with a hazard ratio (HR) of 0.88 (95\% confidence interval (CI) $0.75-1.04$ ) (Malthaner et al, 2006).

The preoperative chemotherapy approach to localised oesophageal cancer may be optimised by the incorporation of more effective cytotoxic agents. The epirubicin/cisplatin/fluorouracil (ECF) triplet regimen is effective in the treatment of advanced gastro-oesophageal cancer (Webb et al, 1997; Ross et al, 2002) and when given as part of a peri-operative strategy for the treatment of localised gastric and lower oesophageal/GOJ adenocarcinoma, resulting in a significant improvement in 5-year survival compared with surgery alone (36 vs 23\%) (Cunningham et al, 2006). Recently, capecitabine $(\mathrm{X})$ and oxaliplatin $(\mathrm{O})$ were found to be as effective as fluorouracil and cisplatin, respectively, when substituted in the ECF regimen for treating advanced gastro-oesophageal cancer (Cunningham et al, 2008). The epirubicin/cisplatin/capecitabine (ECX) and EOX regimens provide more convenient treatment alternatives to ECF (no requirement for an indwelling central venous access device or hydration for oxaliplatin) with certain toxicity advantages.

In patients with oesophageal adenocarcinoma long-term survival is strongly correlated with the achievement of pathological complete response (pCR) to neoadjuvant chemoradiotherapy (Walsh et al, 1996; Geh et al, 2001; Urba et al, 2001; Berger et al, 2005; Burmeister et al, 2005; Rohatgi et al, 2005). However, in the US Intergroup and OEO 2 trials of preoperative chemotherapy, the pCR rates were only 2 and 4\%, respectively (Kelsen et al, 1998; MRC, 2002). With the emergence of more effective combination chemotherapy regimens there is potential to improve preoperative chemotherapy-associated pCR rates and thus survival. As a potential surrogate for outcome, pCR could facilitate the reporting of results from phase II trials, OS being the preferred outcome measure for phase III trials.

The aim of this single centre phase II study was to assess the pCR rate associated with preoperative ECX in patients with localised, operable oesophageal and GOJ adenocarcinoma and to evaluate OS, progression-free survival (PFS), toxicity and patterns of treatment failure.

\section{METHODS}

\section{Patients}

Eligible patients were older than 18 years, had histologically proven adenocarcinoma of the thoracic oesophagus or GOJ (Siewert's types I-III), American Joint Committee on Cancer (AJCC) stage II or III (T2-3, N0-1, M0) disease, an Eastern Cooperative Group (ECOG) performance status $\leqslant 2$ and adequate renal, hepatic and bone marrow function. Exclusion criteria included locally advanced (T4) or metastatic disease (including M1a), prior chemotherapy or radiotherapy, any clinically significant uncontrolled co-existing illness or previous malignant disease and complete dysphagia. This single institution trial was approved by the local Ethics and Scientific Review Committees and all patients provided written informed consent.

\section{Pre-treatment evaluation}

Patients underwent staging computed tomography (CT) of the chest/abdomen/pelvis and endoscopic ultrasound (EUS) within 28 days of the first treatment. Laparoscopy and positron emission tomography (PET) were performed where indicated. Glomerular filtration rate was determined by $24 \mathrm{~h}$ urinary clearance or EDTA testing. Audiography and echocardiography or multiple-gated acquisition scanning were performed when clinically indicated.
Operability was determined by a multi-disciplinary team following this evaluation.

\section{Treatment}

Preoperative chemotherapy Chemotherapy was administered for four cycles preoperatively. Each cycle comprised epirubicin $\left(50 \mathrm{mg} \mathrm{m}^{-2}\right)$ on day 1 by intravenous bolus, cisplatin $\left(60 \mathrm{mg} \mathrm{m}^{-2}\right)$ intravenously on day 1 with pre- and post-hydration and oral capecitabine $\left(625 \mathrm{mg} \mathrm{m}^{-2}\right.$ twice daily) continuously throughout treatment. Before each cycle of chemotherapy, a full blood count and biochemical function (including renal and liver parameters) were checked. Adverse events were assessed at every treatment visit and were graded according to the National Cancer Institute Common Toxicity Criteria (NCI-CTC) version 2.0. Dose modifications were instituted as previously described (Sumpter et al, 2005). Cisplatin was discontinued in the presence of clinically significant ototoxicity or peripheral neuropathy and substituted by carboplatin at the discretion of the investigator. Epirubicin was omitted if there was a clinically significant deterioration in cardiac function confirmed by echocardiography (although cumulative dose only $200 \mathrm{mg} \mathrm{m}^{-2}$ ).

Surgery Post-chemotherapy evaluation included repeat CT chest/ abdomen/pelvis and EUS reviewed by the multi-disciplinary team. Patients with stable or responding disease who remained fit for surgery proceeded to surgery 4-6 weeks after the last cycle of chemotherapy. Surgery was undertaken within one regional highvolume centre (the majority by one surgeon) and was determined by the location and extent of the localised tumour. Patients with Siewert type I or II junctional cancers were treated by right thoraco-abdominal oesophago-gastrectomy with two field lymph node dissection. Those with type III tumours underwent extended total gastrectomy with D2 lymphadenectomy using either a transhiatal approach or a left thoraco-abdominal approach. Resection of other organs was only included if there was evidence of local infiltration. Post-operative complications, and any deaths following surgery were recorded.

Patients deemed inoperable due to progression with locoregional disease were considered for definitive chemoradiation where appropriate. Patients deemed inoperable at surgery due to metastatic disease were followed up and treated according to the local protocols.

\section{Post-treatment evaluation and follow-up}

Resected tumours were reviewed for histopathological response by one histopathologist according to TMN staging after neoadjuvant chemotherapy. The specimens were inflated and pinned to reduce shrinkage of the oesophagus, sliced perpendicular to the lumen in their entirety to give $5 \mathrm{~mm}$ slices and each slice was embedded and processed for histological examination. The whole length of the tumour was examined to exclude the possibility of missing small foci of residual tumour. Tumours were examined for pCR defined as the inability of the pathologist to identify viable malignant cells within the resected specimen. All patients had a post-surgery baseline CT scan and were followed up at 3 monthly intervals for the first year, 6 monthly intervals for the second year and annually thereafter. CT scans (chest/abdomen/pelvis) were routinely performed at 1 and 2 years after surgery and otherwise when clinically indicated.

\section{Statistical analysis}

The primary end point was the $\mathrm{pCR}$ rate in the intent-to-treat population. A Simon's two-stage design (Chen and Shan, 2008) was used based on a lower limit of a pCR rate of $5 \%\left(\mathrm{p}_{0}\right)$ and an acceptable pCR rate of $15 \%\left(\mathrm{p}_{1}\right)$. With a calculated sample size of 
33 patients, the study would stop early if a response rate of lower than $5 \%$ was observed with $80.1 \%$ power $(\alpha=0.049)$. Thus, if $\leqslant 2$ pCRs were observed, the study would terminate due to lack of efficacy, conversely if $\geqslant 6$ pCRs were observed, then a conclusion of efficacy would be reached and the trial stopped. If neither criteria was met, the study would proceed to the second stage, recruiting an additional 20 patients (total $n=53$ ), concluding efficacy only if $\geqslant 6$ pCRs were observed. Seven additional patients could be recruited to address the potential dropouts.

Secondary end points included OS and PFS, objective response rate by $\mathrm{CT}$ and EUS (EUS response was considered to be $>30 \%$ reduction in maximal tumour thickness), toxicity and patterns of treatment failure. Overall survival was calculated from the date of study registration to the date of death from any cause. Progression-free survival was calculated from the date of study registration to the date of first documented progression or death. Patients remaining alive or lost to follow-up were censored at the date of last follow-up. Survival was calculated according to the KaplanMeier method.

\section{RESULTS}

\section{Patients}

Between November 2002 and June 200734 patients were recruited, all eligible for the study. The median age was 60 years (range $41-$ 81 years), most patients were of performance status $0 / 1$ and the majority had T3 disease (79.4\%) with nodal involvement as assessed by EUS. Patient characteristics are indicated in Table 1.

\section{Treatment}

Chemotherapy The median number of preoperative cycles of chemotherapy delivered was 4 . Thirty-one patients completed all

Table I Patient characteristics

\begin{tabular}{lc}
\hline & $\mathbf{N}=\mathbf{3 4}$ \\
\hline Median age, years (range) & $60(4|-8|)$ \\
Sex & \\
Male & $31(91 \%)$ \\
Female & $3(9 \%)$ \\
ECOG performance status & \\
0 & $9(26 \%)$ \\
I & $24(71 \%)$ \\
2 & $1(3 \%)$ \\
Histology & \\
Adenocarcinoma & $34(100 \%)$ \\
Siewert's classification & \\
Type I & $18(53 \%)$ \\
Type II & $12(35 \%)$ \\
Type III & $4(12 \%)$ \\
T stage & \\
T2 & $7(21 \%)$ \\
T3 & $27(79 \%)$ \\
N Stage & \\
No & \\
NI & $11(32 \%)$ \\
N2 & $23(68 \%)$ \\
M stage & $0(0 \%)$ \\
M0 & \\
\hline
\end{tabular}

four cycles. Two patients received only 1 and 3 cycles, respectively, due to progressive disease on therapy as assessed by CT, and one patient received three cycles due to toxicity. For the cohort of 34 patients, the mean cumulative doses of epirubicin, cisplatin and capecitabine were 181, 221 and $93 \mathrm{~g} \mathrm{~m}^{-2}$, respectively (i.e., 90, 92 and $89 \%$ of the planned cumulative doses, respectively). By EUS, 13 of the 28 assessable patients had a response and 15 patients had stable disease.

Surgery Twenty-six patients were resected $(76 \%$ of the 34 patients) after preoperative chemotherapy. One additional resected patient did not have protocol-specified preoperative therapy and was excluded from the resection analysis (considered inoperable after four cycles of chemotherapy, but resected after a subsequent course of chemoradiation). Reasons for not proceeding to resection included progressive disease during preoperative chemotherapy $(n=2)$, inoperable at laparotomy due to peritoneal/liver metastases undetected by preoperative imaging $(n=4)$ and tumour considered too bulky on preoperative imaging $(n=1)$. Of the 26 resected patients, 21 patients underwent oesophagogastrectomy and 6 patients underwent extended total gastrectomy. Nineteen patients achieved R0 resections (no evidence of residual macroscopic or microscopic disease at margins) (73\%; 95\% CI $56-90$ or $56 \%$ on an intent-to-treat basis) and seven had R1 resections by virtue of microscopic disease within $1 \mathrm{~mm}$ of the circumferential resection margins. Of the seven patients who had R1 resections, four received radical post-operative chemoradiotherapy.

\section{Pathological findings}

In the group of 26 resected patients, pCR was seen in 2 patients which represents a pCR rate of $7.4 \%(95 \%$ CI $0-17)$ or $5.9 \%(95 \%$ CI $0-14)$ in the intent-to-treat population, which met the criteria for early study termination due to lack of treatment efficacy based on pCR. Twelve patients experienced pathological T downstaging compared with the initial EUS T stage and five of these were EUS responders.

\section{Survival}

With a median follow-up of 34 months, 12 patients remain alive (9 disease-free). For the 34 patients in the intent-to-treat population, the median OS is 17 months $(95 \%$ CI $12-22)$ and 1 and 2-year survivals are 67\% (95\% CI $48-80 \%)$ and 39\% (95\% CI $23-55 \%)$, respectively (Figure $1 \mathrm{~A})$. The median PFS is 13 months (95\% CI 11-16), Figure 1B. Overall survival according to $\mathrm{R}$ resection status for the 26 resected patients is shown in Figure 2 and indicates a nonsignificant trend for improved survival in the R0 vs R1 group (HR 2.1; 95\% CI 0.7-7.0; $P=0.2$ ). Both patients who achieved pCR developed systemic recurrent disease within 1 year (one with brain metastases and the other with peritoneal disease). In an exploratory analysis, OS according to EUS response was performed (Figure 3) and indicates a nonsignificant trend for improved survival for EUS responders compared with non-responders (HR 2.0; 95\% CI 0.9-5.1; $P=0.2$ ). Eleven patients proceeded to receive systemic chemotherapy for advanced disease.

\section{Chemotherapy-related toxicity and surgical complications}

Adverse events that occurred during chemotherapy are documented in Table 2. There were no chemotherapy-related deaths during preoperative chemotherapy. One patient who received only one cycle of chemotherapy died within 30 days of chemotherapy of objectively documented progressive disease. One patient died after 54 days of surgery with a chronic history of chest problems complicated by surgery and one other died after 31 days due 


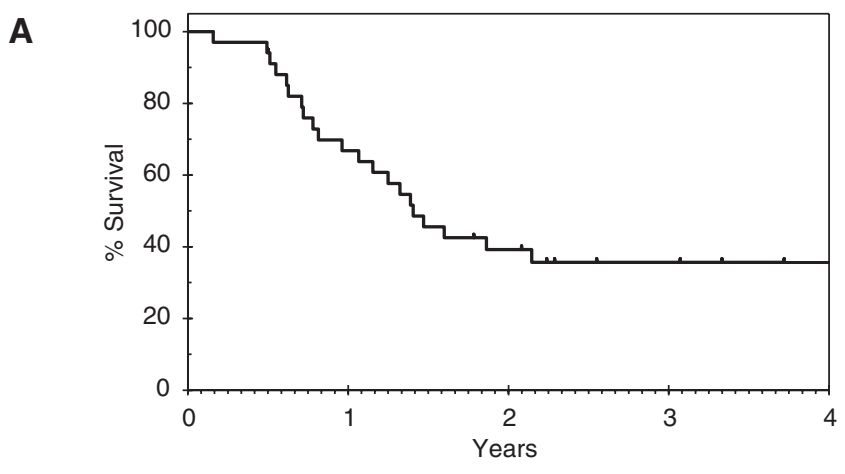

Number at risk 34

12



Number at risk 34

5
Figure I Overall and progression-free survival in the intent-to-treat population. (A) Overall survival. (B) progression-free survival.



Figure 2 Overall survival according to $\mathrm{R}$ resection status $(N=26)$.

to post-operative chylous leak complicated by renal failure (malignant cells were identified in the chylous fluid). Postoperative complications occurring in greater than $5 \%$ of patients included respiratory complications $(n=5)$, chylous leak $(n=2)$, recurrent laryngeal nerve palsy $(n=2)$ and renal failure $(n=2)$. For the 26 patients undergoing resection, first sites of recurrence are indicated in Table 3.

\section{DISCUSSION}

In this phase II study of 34 patients with operable gastrooesophageal adenocarcinoma, preoperative ECX was associated

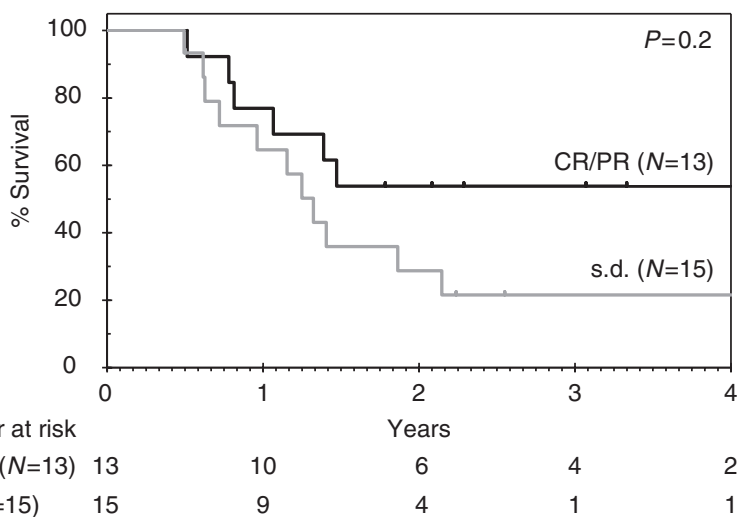

Figure 3 Overall survival according to EUS response $(N=28)$ - on the basis of evaluable patients for whom measurement of maximal tumour thickness was available pre- and post-chemotherapy. Reasons for patients being non-evaluable included death from progressive disease $(n=1)$, stenotic lesion $(n=1)$, EUS data unavailable $(n=2)$, thickness not recorded on second EUS $(n=2)$.

Table 2 Adverse events during preoperative chemotherapy $(N=34)$

\begin{tabular}{|c|c|c|}
\hline & All grades, $n$ (\%) & Grade 3/4, n (\%) \\
\hline \multicolumn{3}{|l|}{ Haematological } \\
\hline Neutropenia & $32(94)$ & $21(62)$ \\
\hline Anaemia & $26(76)$ & $3(9)$ \\
\hline Thrombocytopenia & $8(24)$ & $0(0)$ \\
\hline Febrile neutropenia & I (3) & I (3) \\
\hline \multicolumn{3}{|l|}{ Gastrointestinal } \\
\hline Nausea and vomiting & $26(76)$ & $3(9)$ \\
\hline Diarrhoea & $15(44)$ & I (3) \\
\hline Stomatitis & $13(38)$ & $0(0)$ \\
\hline \multicolumn{3}{|l|}{ Skin } \\
\hline Hand-foot syndrome & $22(64)$ & $5(15)$ \\
\hline \multicolumn{3}{|l|}{ Neurological } \\
\hline Peripheral neuropathy & $8(24)$ & I (3) \\
\hline \multicolumn{3}{|l|}{ Constitutional } \\
\hline Fatigue & $34(100)$ & I (3) \\
\hline
\end{tabular}

Table 3 First sites of recurrence for patients undergoing resection $(N=26)$ (documented relapse, $N=14$ )

\begin{tabular}{lcc}
\hline & \multicolumn{2}{c}{ Number of patients } \\
\cline { 2 - 3 } Site of recurrence & $\mathbf{R 0 ,} \mathbf{N}=\mathbf{1 9}$ & $\mathbf{R} \mathbf{I}, \mathbf{N}=\mathbf{7}$ \\
\hline Local - (resection bed/anastomosis/ & 1 & 3 \\
local lymph nodes) & 5 & 3 \\
Distant & & \\
Local and distant & 2 & 0 \\
No recurrence & 9 & 0 \\
Death without documented recurrence & 2 & 1 \\
\hline
\end{tabular}

${ }^{a}$ Four patients showed recurrence at more than one site. Sites of distant recurrence included distant nodes $(N=6)$, peritoneum $(N=3)$, lung $(N=2)$, brain $(N=1)$, liver $(N=1)$ and bone $(N=1)$

with a pCR rate of only $5.9 \%$ in the intent-to-treat population. This resulted in early termination of the study based on the statistical premise of the trial, which used pCR as the primary end point. This 
raises doubts over whether more effective chemotherapy regimens lead to improved pCR rates compared with those observed in trials of preoperative cisplatin/5FU (Kelsen et al, 1998; MRC, 2002) and highlights the potential limitations of $\mathrm{pCR}$ as a primary end point and surrogate marker of outcome for preoperative chemotherapy. Paradoxically, both patients who achieved pCRs in this study relapsed within 1 year of surgery. The early treatment and potential elimination of micro-metastatic disease may actually be the critical factor in conferring a survival advantage to systemic neo-adjuvant therapy for localised oesophageal cancer. Alternative regimens, for instance those incorporating docetaxel, may result in higher pCR rates in phase II evaluation (Lorenzen et al, 2007) but whether this translates into improved survival has yet to be shown. The median and 2 year survivals of 17 months and $39 \%$, respectively, are comparable to those observed in the MRC and Intergroup trials of preoperative cispatin/fluorouracil (Kelsen et al, 1998; MRC, 2002). However, our study was not powered around survival, included patients mainly with T3 N1 disease and excluded squamous carcinoma, which may account for the reason for survival that did not appear to be greater with an effective triplet regimen. Another consideration is the accuracy of histological assessment of pCR, particularly when used as a primary end point. However, standard histopathological review protocols are likely to be accurate in this regard (Chang et al, 2007), and in our institution all histopathological examination was performed by a single experienced gastrointestinal pathologist. There are no other validated scores of tumour regression in response to preoperative therapy in oesophageal cancer, although recently one group defined responders and non-responders as those with $\leqslant$ or $>10 \%$ residual tumour cells, respectively, in the resected specimen (Weber et al, 2001; Brucher et al, 2006; Ott et al, 2006), and thus pCR remains the standard assessment of histopathological response.

The PCR rates associated with preoperative chemoradiation, the other major treatment strategy for localised oesophageal carcinoma commonly used in North America, range from 15 to $33 \%$ for the randomised controlled trials, which included patients with adenocarcinoma (Walsh et al, 1996; Urba et al, 2001; Burmeister et al, 2005; Tepper et al, 2008). Although higher than those achieved with preoperative chemotherapy, these pCR rates have failed to translate into a consistent survival advantage for preoperative chemoradiation, the two positive studies being criticised for unusually poor survival in the surgery alone arm (Walsh et al, 1996) and for early termination due to poor recruitment (Tepper et al, 2008). A recent meta-analysis of trials of preoperative therapy in localised oesophageal carcinoma showed a similar survival advantage for the subgroup of patients with adenocarcinoma treated with chemoradiation $(n=345$, HR 0.75 , $95 \%$ CI $0.59-0.95 ; P=0.02)$ and chemotherapy alone $(n=533$, HR $0.78,95 \%$ CI $0.64-0.95 ; \quad P=0.014$ ) (Gebski et al, 2007). Notably, there appeared to be a survival advantage in the pooled analysis for squamous carcinoma treated with preoperative chemoradiation but not preoperative chemotherapy, in contrast to observations from subgroup analysis by histology of the MRC trial (Allum et al, 2008). Differential treatment effects have earlier been noted according to histology underscoring that squamous carcinoma and adenocarcinoma are separate clinical and biological entities.

The toxicity associated with preoperative ECX was reasonably consistent with that reported for the regimen in the treatment of advanced disease (Cunningham et al, 2008). The $\mathrm{R} 0$ resection rate of $73 \%$ is higher than that reported in the MRC and Intergroup trials (60 and 63\%, respectively) (MRC, 2002; Kelsen et al, 1998) and the frequency of post-operative complications was low. Subgroup analysis in both the MRC and Intergroup trials indicated that $\mathrm{R} 0$ resection was associated with significantly better survival as compared with $\mathrm{R} 1$ resection or less with a 3-year OS of $42 v s$
$18 \%$ (Allum et al, 2008) and 39 vs 12\% (Kelsen et al, 2007) for R0 vs R1 resection, respectively. In this study, there was a trend towards improved survival for patients achieving an R0 compared with an $\mathrm{R} 1$ resection, which did not reach statistical significance and is likely to reflect the small patient numbers. The majority of patients relapsing after resection presented with distant metastases, consistent with earlier observations in oesophageal and GOJ adenocarcinoma (Wayman et al, 2002), and highlighting the need for surgical adjuncts incorporating effective systemically directed therapies in this disease. Preoperative approaches remain the most extensively investigated with only a limited number of small negative randomised trials assessing adjuvant therapy mainly in squamous carcinoma (Malthaner et al, 2004).

Accurate assessment of response to preoperative therapy and its correlation with outcomes may facilitate the rationalisation of further treatment, limit surgery-related morbidity and mortality and optimise outcomes. As compared with CT and oesophagogastroscopy, EUS is more reliable for determining response to preoperative therapy (Brown et al, 2004; Westerterp et al, 2005). In an exploratory analysis, we attempted to assess the potential of EUS as a tool for outcome prediction to preoperative chemotherapy, evaluating the change in maximal tumour thickness with a decrease of $\geqslant 30 \%$ representing a response, the cut-off value utilised in RECIST and a simple response classifier. Response according to EUS and its correlation with outcomes, such as pathological response and survival, has previously been shown in small studies of neoadjuvant therapy for oesophageal cancer (Hirata et al, 1997; Chak et al, 2000; Willis et al, 2002; Masaho et al, 2005). In our analysis, there was no difference in OS between EUS responders and non-responders to preoperative chemotherapy and is likely to reflect the small number of patients. In addition, EUS is limited in its inability to distinguish post-treatment fibrosis and inflammation from tumour (Lightdale and Kulkarni, 2005). A more promising imaging biomarker for outcome prediction to preoperative chemotherapy further along in evaluation is metabolic response by PET. Having been identified retrospectively (Weber et al, 2001) and validated prospectively (Ott et al, 2006), the feasibility of an early metabolic response-adjusted treatment algorithm has been confirmed in a non-randomised trial (Lordick et al, 2007), and randomised assessment of this biomarker-driven treatment strategy is planned. There is also currently extensive investigation to identify predictive and prognostic molecular markers and signatures.

Efforts to improve the preoperative chemotherapy approach to localised oesophageal cancer include the incorporation of newer more effective cytotoxics including the taxanes, oxaliplatin and capecitabine and targeted agents such as bevacizumab. In an ongoing UK phase III trial of the MRC patients with oesophageal/ GOJ (types I and II) adenocarcinoma are randomised between two cycles of cisplatin/fluorouracil and four cycles of ECX with OS as the primary end point. This study was designed as a prelude to the MRC trial, partly to provide some initial safety data on preoperative ECX, as the randomised study of ECF $v s$ the capecitabine and oxaliplatin regimens in the advanced disease setting (Cunningham et al, 2008) had not reported at that time. Many patients at our institution preferentially enrolled in the randomised trial following its launch in 2004. In another ongoing trial of the MRC, patients with gastric and type III GOJ adenocarcinoma are randomised between six cycles of preand post-operative ECX or the same with the addition of bevacizumab. The present phase II trial indicated the safety and feasibility of administering preoperative ECX for oesophageal and GOJ tumours, that preoperative chemotherapy is associated with low pCR rates and the limitations of $\mathrm{pCR}$ as a surrogate marker of outcome for preoperative chemotherapy. 


\section{REFERENCES}

Allum WH, Fogarty PJ, Stenning SP, Langley RE (2008) Long term results of the MRC OEO2 randomized trial of surgery with or without preoperative chemotherapy in resectable esophageal cancer. Proc Gastrointestinal Cancers Symposium. Abstract 9

Berger AC, Farma J, Scott WJ, Freedman G, Weiner L, Cheng JD, Wang H, Goldberg M (2005) Complete response to neoadjuvant chemoradiotherapy in esophageal carcinoma is associated with significantly improved survival. J Clin Oncol 23: $4330-4337$

Blot WJ, Devesa SS, Kneller RW, Fraumeni Jr JF (1991) Rising incidence of adenocarcinoma of the esophagus and gastric cardia. JAMA 265: $1287-1289$

Brown WA, Thomas J, Gotley D, Burmeister BH, Lim KH, Martin I, Walpole ET, Thomson DB, Harvey JA, Smithers BM (2004) Use of oesophagogastroscopy to assess the response of oesophageal carcinoma to neoadjuvant therapy. Br J Surg 91: 199-204

Brucher BL, Becker K, Lordick F, Fink U, Sarbia M, Stein H, Busch R, Zimmermann F, Molls M, Hofler H, Siewert JR (2006) The clinical impact of histopathologic response assessment by residual tumor cell quantification in esophageal squamous cell carcinomas. Cancer 106: 2119-2127

Burmeister BH, Smithers BM, Gebski V, Fitzgerald L, Simes RJ, Devitt P, Ackland S, Gotley DC, Joseph D, Millar J, North J, Walpole ET, Denham JW (2005) Surgery alone versus chemoradiotherapy followed by surgery for resectable cancer of the oesophagus: a randomised controlled phase III trial. Lancet Oncol 6: 659-668

Chak A, Canto MI, Cooper GS, Isenberg G, Willis J, Levitan N, Clayman J, Forastiere A, Heath E, Sivak Jr MV (2000) Endosonographic assessment of multimodality therapy predicts survival of esophageal carcinoma patients. Cancer 88: $1788-1795$

Chang EY, Smith CA, Corless CL, Thomas Jr CR, Hunter JG, Jobe BA (2007) Accuracy of pathologic examination in detection of complete response after chemoradiation for esophageal cancer. Am J Surg 193: 614-617

Chen K, Shan M (2008) Optimal and minimax three-stage designs for phase II oncology clinical trials. Contemp Clin Trials 29: $32-41$

Cunningham D, Allum WH, Stenning SP, Thompson JN, Van de Velde CJ, Nicolson M, Scarffe JH, Lofts FJ, Falk SJ, Iveson TJ, Smith DB, Langley RE, Verma M, Weeden S, Chua YJ, MAGIC TP (2006) Perioperative chemotherapy versus surgery alone for resectable gastroesophageal cancer. $N$ Engl J Med 355: $11-20$

Cunningham D, Starling N, Rao S, Iveson T, Nicolson M, Coxon F, Middleton G, Daniel F, Oates J, Norman AR (2008) Capecitabine and oxaliplatin for advanced esophagogastric cancer. $N$ Engl J Med 358: $36-46$

Gebski V, Burmeister B, Smithers BM, Foo K, Zalcberg J, Simes J (2007) Survival benefits from neoadjuvant chemoradiotherapy or chemotherapy in oesophageal carcinoma: a meta-analysis. Lancet Oncol 8: 226-234

Geh JI, Crellin AM, Glynne-Jones R (2001) Preoperative (neoadjuvant) chemoradiotherapy in oesophageal cancer. Br J Surg 88: 338-356

Hirata N, Kawamoto K, Ueyama T, Masuda K, Utsunomiya T, Kuwano H (1997) Using endosonography to assess the effects of neoadjuvant therapy in patients with advanced esophageal cancer. AJR Am J Roentgenol 169: $485-491$

Kelsen DP, Ginsberg R, Pajak TF, Sheahan DG, Gunderson L, Mortimer J, Estes N, Haller DG, Ajani J, Kocha W, Minsky BD, Roth JA (1998) Chemotherapy followed by surgery compared with surgery alone for localized esophageal cancer. N Engl J Med 339: 1979-1984

Kelsen DP, Winter KA, Gunderson LL, Mortimer J, Estes NC, Haller DG, Ajani JA, Kocha W, Minsky BD, Roth JA, Willett CG (2007) Long-term results of RTOG trial 8911 (USA Intergroup 113): a random assignment trial comparison of chemotherapy followed by surgery compared with surgery alone for esophageal cancer. J Clin Oncol 25: 3719-3725

Lightdale CJ, Kulkarni KG (2005) Role of endoscopic ultrasonography in the staging and follow-up of esophageal cancer. J Clin Oncol 23: $4483-4489$

Lordick F, Ott K, Krause BJ, Weber WA, Becker K, Stein HJ, Lorenzen S, Schuster T, Wieder H, Herrmann K, Bredenkamp R, Hofler H, Fink U, Peschel C, Schwaiger M, Siewert JR (2007) PET to assess early metabolic response and to guide treatment of adenocarcinoma of the oesophagogastric junction: the MUNICON phase II trial. Lancet Oncol 8: 797-805

Lorenzen S, Hentrich M, Haberl C, Heinemann V, Schuster T, Seroneit T, Roethling N, Peschel C, Lordick F (2007) Split-dose docetaxel, cisplatin and leucovorin/fluorouracil as first-line therapy in advanced gastric cancer and adenocarcinoma of the gastroesophageal junction: results of a phase II trial. Ann Oncol 18: $1673-1679$
Malthaner RA, Collin S, Fenlon D (2006) Preoperative chemotherapy for resectable thoracic esophageal cancer. Cochrane Database Syst Rev 3: CD001556

Malthaner RA, Wong RK, Rumble RB, Zuraw L (2004) Neoadjuvant or adjuvant therapy for resectable esophageal cancer: a systematic review and meta-analysis. BMC Med 2: 35

Masaho O, Yoko M, Hiroko Ieal (2005) Useful endoscopic ultrasonography to assess the efficacy of neoadjuvant therapy for advanced esophageal carcinoma: based on the response evaluation criteria in solid tumours. Digestive Endoscopy 17: 59-63

McCann J (1999) Esophageal cancers: changing character, increasing incidence. J Natl Cancer Inst 91: 497-498

MRC (2002) Surgical resection with or without preoperative chemotherapy in oesophageal cancer: a randomised controlled trial. Lancet 359: 1727-1733

Ott K, Weber WA, Lordick F, Becker K, Busch R, Herrmann K, Wieder H, Fink U, Schwaiger M, Siewert JR (2006) Metabolic imaging predicts response, survival, and recurrence in adenocarcinomas of the esophagogastric junction. J Clin Oncol 24: $4692-4698$

Parkin DM, Bray F, Ferlay J, Pisani P (2005) Global cancer statistics, 2002. CA Cancer J Clin 55: 74-108

Pera M, Manterola C, Vidal O, Grande L (2005) Epidemiology of esophageal adenocarcinoma. J Surg Oncol 92: 151 -159

Rohatgi P, Swisher SG, Correa AM, Wu TT, Liao Z, Komaki R, Walsh GL, Vaporciyan AA, Rice DC, Roth JA, Ajani JA (2005) Characterization of pathologic complete response after preoperative chemoradiotherapy in carcinoma of the esophagus and outcome after pathologic complete response. Cancer 104: $2365-2372$

Ross P, Nicolson M, Cunningham D, Valle J, Seymour M, Harper P, Price T, Anderson H, Iveson T, Hickish T, Lofts F, Norman A (2002) Prospective randomized trial comparing mitomycin, cisplatin, and protracted venousinfusion fluorouracil (PVI 5-FU) With epirubicin, cisplatin, and PVI 5-FU in advanced esophagogastric cancer. J Clin Oncol 20: 1996-2004

Sumpter K, Harper-Wynne C, Cunningham D, Rao S, Tebbutt N, Norman AR, Ward C, Iveson T, Nicolson M, Hickish T, Hill M, Oates J (2005) Report of two protocol planned interim analyses in a randomised multicentre phase III study comparing capecitabine with fluorouracil and oxaliplatin with cisplatin in patients with advanced oesophagogastric cancer receiving ECF. Br J Cancer 92: 1976-1983

Tepper J, Krasna MJ, Niedzwiecki D, Hollis D, Reed CE, Goldberg R, Kiel K, Willett C, Sugarbaker D, Mayer R (2008) Phase III trial of trimodality therapy with cisplatin, fluorouracil, radiotherapy, and surgery compared with surgery alone for esophageal cancer: CALGB 9781. J Clin Oncol 26: $1086-1092$

Urba SG, Orringer MB, Turrisi A, Iannettoni M, Forastiere A, Strawderman M (2001) Randomized trial of preoperative chemoradiation versus surgery alone in patients with locoregional esophageal carcinoma. J Clin Oncol 19: $305-313$

Walsh TN, Noonan N, Hollywood D, Kelly A, Keeling N, Hennessy TP (1996) A comparison of multimodal therapy and surgery for esophageal adenocarcinoma. $N$ Engl J Med 335: $462-467$

Wayman J, Bennett MK, Raimes SA, Griffin SM (2002) The pattern of recurrence of adenocarcinoma of the oesophago-gastric junction. $\mathrm{Br} J$ Cancer 86: 1223 - 1229

Webb A, Cunningham D, Scarffe JH, Harper P, Norman A, Joffe JK, Hughes M, Mansi J, Findlay M, Hill A, Oates J, Nicolson M, Hickish T, O’Brien M, Iveson T, Watson M, Underhill C, Wardley A, Meehan M (1997) Randomized trial comparing epirubicin, cisplatin, and fluorouracil versus fluorouracil, doxorubicin, and methotrexate in advanced esophagogastric cancer. J Clin Oncol 15: 261-267

Weber WA, Ott K, Becker K, Dittler HJ, Helmberger H, Avril NE, Meisetschlager G, Busch R, Siewert JR, Schwaiger M, Fink U (2001) Prediction of response to preoperative chemotherapy in adenocarcinomas of the esophagogastric junction by metabolic imaging. J Clin Oncol 19: $3058-3065$

Westerterp M, van Westreenen HL, Reitsma JB, Hoekstra OS, Stoker J, Fockens P, Jager PL, Van Eck-Smit BL, Plukker JT, van Lanschot JJ, Sloof GW (2005) Esophageal cancer: CT, endoscopic US, and FDG PET for assessment of response to neoadjuvant therapy - systematic review. Radiology 236: $841-851$

Willis J, Cooper GS, Isenberg G, Sivak Jr MV, Levitan N, Clayman J, Chak A (2002) Correlation of EUS measurement with pathologic assessment of neoadjuvant therapy response in esophageal carcinoma. Gastrointest Endosc 55: $655-661$ 